\title{
Non-COVID-19 deaths after social distancing in Norway
}

\author{
Ralph Catalano ${ }^{1}$ (1) $\cdot$ Joan A. Casey ${ }^{2} \cdot$ Tim A. Bruckner $^{3} \cdot$ Alison Gemmill $^{4}$
}

Received: 10 June 2020 / Accepted: 30 October 2020 / Published online: 9 November 2020

(c) Springer Nature B.V. 2020

\begin{abstract}
Lay persons and policy makers have speculated on how national differences in the imposition of social distancing to reduce SARS CoV-2 (severe acute respiratory syndrome coronavirus 2) infection has affected non-COVID-19 deaths. No rigorous estimation of the effect appears in the scholarly literature. We use time-series methods to compare non-COVID-19 deaths in Norway during its 9 weeks of mandated social distancing to those expected from history as well as from non-COVID-19 deaths in relatively less restricted Sweden. We estimate that 430 fewer Norwegians than expected died from causes other than COVID-19. We argue that failing to account for averted non-COVID-19 deaths will lead to an underestimate of the benefits of social distancing policies.
\end{abstract}

Keywords Non-Covid-19 Deaths $\cdot$ Social Distancing $\cdot$ Norway

\section{Introduction}

Intuition and limited data $[1,2]$ suggest that social distancing intended to reduce SARS-CoV-2 (severe acute respiratory syndrome coronavirus 2) infections may also affect the incidence of death caused by the hazards of "normal" life. Flu and other infectious diseases as well as work and motor vehicle accidents, for example, may decline when social interactions become less common. Such distancing may, however, also increase deaths by reducing social support as well as by impeding access to medical care. Formal estimation of the "net effect" of these countervailing mechanisms on the incidence of non-COVID-19 deaths would help inform collective choices concerning the management of the

Ralph Catalano

rayc@berkeley.edu

1 School of Public Health, University of California, Berkeley, CA 94720, USA

2 Department of Environmental Health Sciences, Columbia University Mailman School of Public Health, New York, NY, USA

3 Program in Public Health, University of California, Irvine, USA

4 Department of Population, Family and Reproductive Health, Johns Hopkins Bloomberg School of Public Health, Baltimore, MD, USA pandemic [3]. No such estimates, however, appear in the scholarly literature.

On March 12, 2020, Norway's government announced a series of non-pharmaceutical interventions that curtailed social interaction [4]. As widely reported, Sweden's government chose less restrictive policies [5]. These different approaches taken by neighboring countries with similar socio-economic characteristics provide an opportunity to estimate changes in the incidence of death associated with enforced social distancing policies.

We apply time-series methods to compare non-COVID-19 deaths observed in Norway before and during the epidemic to those expected from non-COVID-19 deaths in Sweden as well as from the history of Norwegian mortality trends. We focus, as described below, on the 9 weeks during which Norwegian and Swedish policies diverged. These started Monday, March 16. Most of these policies remained in place, including school closure, until Monday May 18 [6].

\section{Methods}

We obtained weekly counts of deaths for the 540 weeks beginning January 4, 2010 and ending May 17, 2020 from the Human Mortality Database's Short-term Mortality Fluctuations (STMF) data series [7]. Deaths were assigned to weeks based on their date of occurrence. Data from Sweden 
were provided by Eurostat and Statistics Sweden, while data from Norway were provided by Statistics Norway.

We also obtained daily counts of COVID-19 deaths from the Your World in Data Website [8] and aggregated them to weeks. We created our test variables by subtracting COVID19 weekly deaths from all weekly deaths for both Norway and Sweden.

Our analyses used Box-Jenkins time-series methods [9] adapted for epidemiologic questions [10]. These methods remove from weekly Norwegian deaths the variation predictable not only from deaths in Sweden, but also from autocorrelation (i.e., trends, seasonality, or the tendency to remain elevated or depressed after high or low values) observed in the history of Norwegian deaths before social distancing. Box-Jenkins models use parameters for short (i.e., moving average) and long (i.e., autoregressive) "memory" in processes assumed to generate time series. This approach rules out confounding by "third variables" affecting both Sweden and Norway as well as those peculiar to Norway that exhibit autocorrelation. The approach also assures a statistically efficient estimation of the association with the imposition of social distancing because the adjusted series will meet the assumptions of constant mean and serial independence.

We proceeded through 4 steps, described below, using Box-Jenkins time series modeling.

1. We regressed the weekly number of non-COVID-19 deaths in Norway on those in Sweden for the 540 weeks beginning January 4, 2010 and ending May 17, 2020.

2. We used Box and Jenkins methods to detect autocorrelation including trends, cycles (e.g. seasonality), and/or the tendency to remain temporarily elevated or depressed after high or low values in the residuals of the regression estimated in step 1.

3. We estimated a Box-Jenkins transfer function formed by including parameters specifying autocorrelation detected in step 2 in the model estimated in step 1.

4. We estimated a test equation formed by adding a binary "social distancing" variable to the transfer function model developed in step 3. We scored the binary variable 1 for the 9 weeks starting March 16 and ending May 17 and 0 otherwise.

\section{Results}

An average of 786 Norwegians, ranging from 663 to 1048, died from non-COVID-19 related causes per week over the 540 weeks of our study. 1716 Swedes on average died from causes other than COVID-19 each week with a range of 1441 to 2204.

Table 1 shows the estimated transfer functions for deaths among Norwegians for the 540 weeks beginning January 4, 2010 and ending May 17, 2020. As expected, the difference in population size causes the coefficient for Swedish deaths to fall below 1 . As indicated by the autoregressive parameter at 51 weeks, Norwegian deaths exhibit seasonality. The autoregressive and moving average parameters at 1 week suggest "memory" of high or low values of death from one week to the next.

Deaths in the 9 weeks of mandated social distancing fell significantly $(p<.05,2$-tailed test) below expected. The estimated coefficient suggests that approximately 430 fewer Norwegians than expected from deaths in Sweden and from autocorrelation peculiar to Norway died in those 9 weeks (i.e., $-47.8 \times 9)$. These 430 averted deaths represent about $6 \%$ of expected non-COVID-19 deaths (i.e., 7299) had Norway not mandated social distancing for 9 weeks. To add perspective, deaths attributed to COVID-19 during these weeks totaled 230 .

Figure 1 shows our results more graphically. Weekly nonCOVID-19 deaths appear as circles for Norwegians for all 52 weeks of 2019 and the first 20 (i.e., through May 17) of 2020. We plot only these 72 weeks because including all 540 would create a graph with resolution that obscures the information from the 9 weeks of mandated social distancing. The darkened circles show the 9 weeks of mandated social distancing. The solid line through the Norwegian data shows
Table 1 Estimated coefficients and standard errors for transfer functions predicting nonCOVID 19 deaths in Norway and Sweden for 540 weeks beginning January 3, 2010 and ending May 17, 2020

\begin{tabular}{|c|c|c|c|c|}
\hline & \multicolumn{2}{|l|}{ Norway } & \multicolumn{2}{|l|}{ Sweden } \\
\hline & Estimate & SE & Estimate & SE \\
\hline Constant & $311.51^{* *}$ & 36.42 & $1378.08 * *$ & 90.80 \\
\hline Weekly Deaths in Comparison Country & $0.28 * *$ & 0.02 & $0.55 * *$ & 0.08 \\
\hline Mandating Social Distancing & $-47.81 * *$ & 20.07 & $107.33^{*}$ & 61.82 \\
\hline \multicolumn{5}{|l|}{ Box Jenkins Parameters } \\
\hline Moving Average at t-1 & $0.67 * *$ & 0.07 & $0.38 * *$ & 0.05 \\
\hline Autoregression at $\mathrm{t}-1$ & $0.88 * *$ & 0.05 & $0.89 * *$ & 0.03 \\
\hline Autoregression at $\mathrm{t}-51$ & $0.14 * *$ & 0.04 & $0.15 * *$ & 0.05 \\
\hline
\end{tabular}

${ }^{*} \mathrm{p}<.10 ; 2$-tailed test

$* * \mathrm{p}<.05 ; 2$-tailed test 
Fig. 1 Observed and expected values of Norwegian and Swedish non-Covid-19 deaths for 72 weeks from December 31, 2018 through May 17, 2020. Observed values appear as circles for Norway and boxes for Sweden. Circles and boxes during Norwegian mandated social distancing appear filled. The solid and dashed lines are expected values for Norway and Sweden respectively

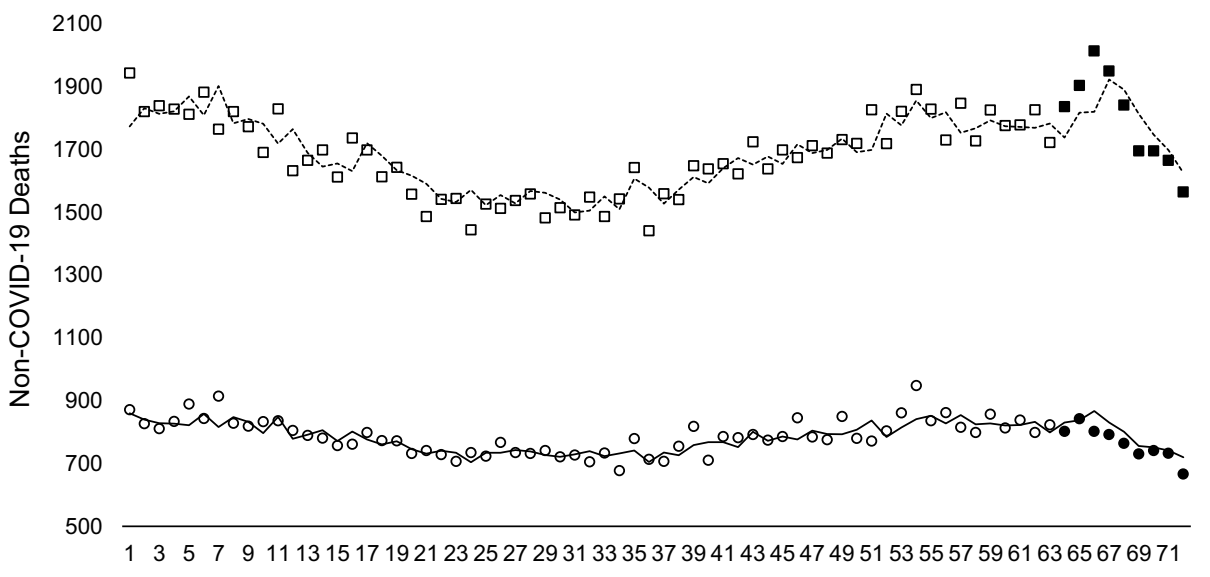

Weeks Starting December 31, 2018 and Ending May 17, 2020 the values expected from non-COVID-19 deaths in Sweden and from autocorrelation peculiar to Norway. As implied by the statistical finding of 430 fewer than expected deaths during mandated social distancing, the values for the last 9 weeks fall predominantly below expected.

Our findings for Norway do not axiomatically imply that Sweden suffered more non-COVID-19 deaths than expected from autocorrelation and similar deaths in Norway during Norwegian social distancing. To determine what transpired in Sweden, we repeated our test but transposed Sweden and Norway. The results of the statistical test appear in Table 1 which shows that about 963 (i.e., 107.3×9) more Swedes than expected died from non-COVID-19 causes during the 9 weeks. Unlike the results for Norway in which the difference appeared detectable at $p<.05$ (2-tailed test), the difference for Sweden were detected at $p<.10$. These results appear more graphically in Fig. 1 in which observed values for Sweden during the mandated social distancing months in Norway (i.e., the filled boxes) appear above the expected values, shown by the dashed line, in the earliest of the 9 weeks and regress to expected later in the series.

\section{Discussion}

We find that approximately 430 fewer Norwegians than expected died from the hazards of normal life in the 9 weeks of imposed social distancing. We derived expected deaths from those in Sweden and from patterns in the history of death in Norway. We do not have cause of death data for the test period so we cannot test speculation as to which hazards declined. Given that social distancing interventions presumably reduce the spread of infection, we speculate that many of the averted deaths would have arisen from infectious diseases. Avoidance of work and recreation and the transportation associated with both implies that accidental deaths also likely fell below expected. We cannot rule out that iatrogenic deaths, particularly those from healthcare-acquired infections, also declined. In Norway, as elsewhere, non-essential use of hospital services fell during the COVID-19 epidemic [11] thereby leaving fewer persons at risk of healthcareacquired infections and death [12].

We do not have weekly COVID-19 deaths by age and gender and so cannot estimate which demographic groups averted the most non-COVID-19 deaths. Whether the age distribution of all deaths predicts the age distribution of averted deaths during mandated social distancing therefore remains unknown.

Strengths of our study include the consistency of methods used to report all-cause mortality in these two Scandinavian countries over a long period. The divergence of policies at a discrete time between these adjacent countries, with otherwise similar economic and social policies, further "borrows strength" in estimating lives saved in Norway by social distancing. This circumstance permits the use of rigorous time-series methods to derive counterfactual (i.e., expected) values of weekly mortality in Norway had the government not imposed social distancing.

We cannot control for all phenomena that plausibly induce weekly variation in Norwegian non-COVID-19 deaths. The comparison population design that we employ does, however, control any such phenomena that also affect variation in Swedish deaths such as seasonal temperature changes. Mortality displacement_or "harvesting"-also appears unlikely to account for our findings as both Swedes and Norwegians would have been subject to this temporal shift in deaths due to COVID-19. Our use of Box-Jenkins modeling, moreover, controls for any phenomena peculiar to Norway that induce regular patterns (i.e, autocorrelation) in weekly Norwegian deaths. But phenomena unique to Norway that exhibit no autocorrelation (e.g., acute local weather extremes, changes in record keeping rules) remain uncontrolled. We argue that these phenomena unlikely pose parsimonious alternatives to our inference that mandated 
social distancing reduced non-COVID-19 deaths in Norway. To rival that inference, such circumstances would have to coincide exactly with mandated social distancing in Norway.

Although not compelled to distance themselves by law, Swedes likely did much to reduce their chances of infection. Using Sweden to generate counterfactual (i.e., expected) values for Norway could, therefore, have biased our estimates towards the null. We may have, as a result, underestimated the true effect of social distancing policies on non-COVID19-related deaths in Norway.

We focused on Norway and Sweden to gain internal validity for our test. We have, however, no empirical estimate of the external validity of our findings. Further research should estimate the effects of other nations' policies in response to COVID-19 on mortality in the short and long term [e.g., 2]. Delayed care-seeking in response to perceived COVID-19 risk may, for example, decrease iatrogenic deaths in the short run but increase mortality over time. Based on estimates of seasonality in COVID-19 infections and deaths, such analyses will likely require collection of data well into 2021 . More in-depth estimates of the "net effect" of these national measures on other population health indicators (e.g., disability adjusted life years) should prove useful in the debate over the efficacy and prudence of various COVID-19 public health policies [3].

\section{References}

1. Baud D, Qi X, Nielsen-Saines K, Musso D, Pomar L, Favre G. Real estimates of mortality following COVID-19 infection. Lancet Infect Dis. 2020;20:20. https://doi.org/10.1016/s1473 -3099(20)30195-X.

2. Mills EHA, Møller AL, Gnesin F, Zylyftari N, Broccia M, Jensen B, Schou M, Fosbøl EL, Køber L, Andersen MP, Phelps M, Gerds T, Torp-Pedersen C. National all-cause mortality during the COVID-19 pandemic: a Danish registry-based study. Eur J
Epidemiol. 2020;21:1-13. https://doi.org/10.1007/s10654-02000680-x.

3. Lenzer J. Covid-19 Group of UK and US experts argues for "focused protection" instead of lockdowns. BMJ. 2020. https:// doi.org/10.1136/bmj.m3908.

4. Norwegian Directorate of Health. The Directorate of Health has adopted comprehensive measures to prevent the spread of Covid19. https://www.helsedirektoratet.no/nyheter/helsedirektoratethar-vedtatt-omfattende-tiltak-for-a-hindre-spredning-av-covid -19. Accessed 26 May 2020.

5. Government Offices of Sweden. The Government's work in response to the virus responsible for COVID-19. https://www. government.se/government-policy/the-governments-work-inresponse-to-the-virus-responsible-for-covid-19/. Accessed 26 May 2020.

6. Office of Norwegian Prime Minister. The Government's plan for reopening Norwegian society and easing the coronavirus restrictions. https://www.regjeringen.no/en/aktuelt/the-government s-plan-for-reopening-norwegian-society-and-easing-the-coron avirus-restrictions/id2701493/. Accessed 26 May 2020.

7. Human Mortality Database. Short-term Mortality Fluctuations (STMF) data series. https://www.mortality.org/. Accessed 27 May 2020.

8. Ritchie, H. Coronavirus Source Data. Our World in Data. https ://ourworldindata.org/coronavirus-source-data. Accessed 4 June 2020.

9. Box GE, Jenkins GM, Reinsel GC, Ljung GM. Time series analysis: forecasting and control. New York: Wiley; 2015.

10. Catalano R, Serxner S. Time series designs of potential interest to epidemiologists. Am J Epidemiol. 1987;126:724-31.

11. Lindeman RJ, Sund M, Löfgren J, Basso T, Søreide K. Preventing spread of SARS-CoV-2 and preparing for the COVID-19 outbreak in the surgical department: perspectives from two Scandinavian countries. J Surg Case Rep. 2020. https://doi.org/10.1093/jscr/ rjaa131.

12. Bjark $\mathrm{PH}$, Hansen $\mathrm{E}$, Lingaas $\mathrm{E}$. In-hospital deaths attributable to healthcare-associated infections. Tidsskr Nor Laegeforen. 2020. https://doi.org/10.4045/tidsskr.19.0288.

Publisher's Note Springer Nature remains neutral with regard to jurisdictional claims in published maps and institutional affiliations. 